\title{
Spin Control Without Magnetic Fields
}

\author{
Researchers demonstrate that they can control the polarization direction \\ of a spin current without having to apply a magnetic field, which could aid \\ in implementing energy-efficient spintronics devices.
}

\section{By Katherine Wright}

$\mathrm{n}$ graphene, spin currents can live much

longer than they can in other materials, making the material an ideal platform for future spintronic devices. But there is a problem: To manipulate graphene's spin currents, researchers need to apply a magnetic field to the material. The necessary hardware is difficult to integrate into circuits, limiting how small graphene-based spin devices could be shrunk. Now Josep Ingla-Aynés of the Nanoscience Cooperative Research Center (CIC NanoGUNE), Spain, and colleagues have demonstrated a method to manipulate-at room temperature-graphene spin currents using only electric fields [1].

The team stamped a sheet of tungsten diselenide on a sheet of bilayer graphene and heated the two materials to bond them together. Then they patterned the structure with a series of electrodes, which they used to apply an in-plane electric field and a gate voltage and to inject a spin current into the graphene. Experiments were performed at $50 \mathrm{~K}$ and at room temperature.

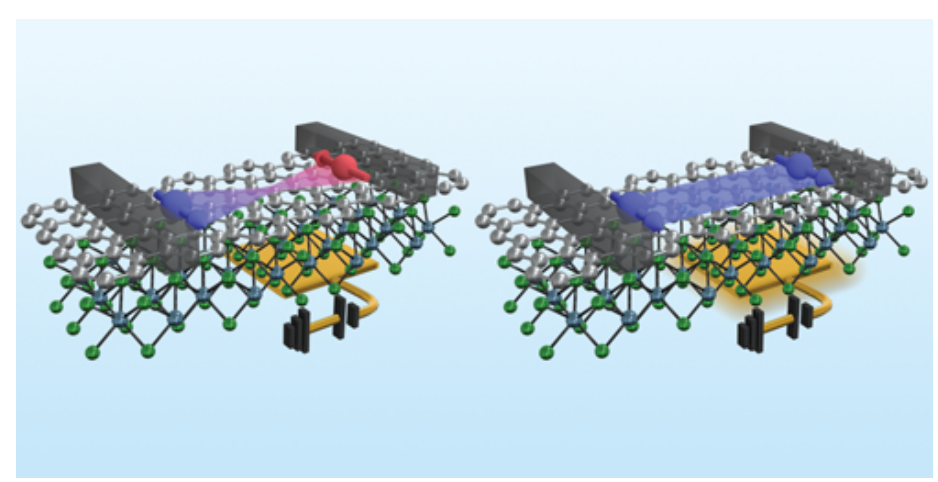

Credit: J. Ingla-Aynés et al. [1]
At both temperatures, the team observed that they could switch the polarization direction of the spin current by changing the magnitude of both the in-plane electric field and the gate voltage. They say that the control comes from the presence of spin-orbit coupling in the tungsten diselenide layer. This effect produces an effective magnetic field in the graphene that is sufficient to change the spin angle.

Ingla-Aynés says that the demonstration represents a room-temperature version of the long-sought-after "Datta Das" spin transistor. The Datta Das spin transistor is a device whose electrical resistance can be switched from high to low by changing the polarization direction of the spin current. Such devices have been realized at low temperatures using two-dimensional electron gases but not at higher temperatures.

Katherine Wright is the Deputy Editor of Physics.

\section{REFERENCES}

1. J. Ingla-Aynés et al., "Electrical control of valley-Zeeman spin-orbit-coupling-induced spin precession at room temperature," Physical Review Letters 127, 047202 (2021). 